\title{
ARTICLES
}

\section{Strategies for containing an emerging influenza pandemic in Southeast Asia}

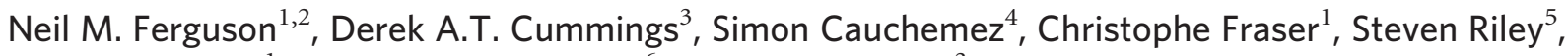 \\ Aronrag Meeyai ${ }^{1}$, Sopon lamsirithaworn ${ }^{6} \&$ Donald S. Burke ${ }^{3}$
}

\begin{abstract}
Highly pathogenic H5N1 influenza A viruses are now endemic in avian populations in Southeast Asia, and human cases continue to accumulate. Although currently incapable of sustained human-to-human transmission, H5N1 represents a serious pandemic threat owing to the risk of a mutation or reassortment generating a virus with increased transmissibility. Identifying public health interventions that might be able to halt a pandemic in its earliest stages is therefore a priority. Here we use a simulation model of influenza transmission in Southeast Asia to evaluate the potential effectiveness of targeted mass prophylactic use of antiviral drugs as a containment strategy. Other interventions aimed at reducing population contact rates are also examined as reinforcements to an antiviral-based containment policy. We show that elimination of a nascent pandemic may be feasible using a combination of geographically targeted prophylaxis and social distancing measures, if the basic reproduction number of the new virus is below 1.8 . We predict that a stockpile of 3 million courses of antiviral drugs should be sufficient for elimination. Policy effectiveness depends critically on how quickly clinical cases are diagnosed and the speed with which antiviral drugs can be distributed.
\end{abstract}

The continuing spread of H5N1 highly pathogenic avian influenza in wild and domestic poultry in Southeast Asia represents the most serious human pandemic influenza risk for decades ${ }^{1,2}$. Great potential benefits would be gained from any intervention able to contain the spread of a pandemic strain and eliminate it from the human population. However, the rapid rate of spread of influenza-as witnessed both in annual epidemics and past pandemics ${ }^{3-5}$ - poses a significant challenge to the design of a realistic control strategy.

The basic reproduction number ${ }^{6}, R_{0}$, quantifies the transmissibility of any pathogen, which is defined as the average number of secondary cases generated by a typical primary case in an entirely susceptible population. A disease can spread if $R_{0}>1$, but if $R_{0}<1$, chains of transmission will inevitably die out. Hence, the goal of control policies is to reduce $R_{0}$ to below 1 by eliminating a proportion $1-1 / R_{0}$ of transmission. This can be achieved in three ways: (1) by reducing contact rates in the population (through 'social distance measures'), (2) by reducing the infectiousness of infected individuals (through treatment or isolation), or (3) by reducing the susceptibility of uninfected individuals (by vaccination or antiviral prophylaxis).

Vaccination and antiviral drugs offer protection against infection and clinical disease. However, although effective vaccines exist for interpandemic flu, candidate $\mathrm{H} 5 \mathrm{~N} 1$ vaccines have unproven effectiveness ${ }^{7}$, and production delays would in any case limit availability in the first months of a pandemic. Antiviral agents-particularly the neuraminidase inhibitors, which show experimental effectiveness against all influenza A subtypes ${ }^{8,9}$-are therefore a key aspect of recently revised pandemic preparedness plans in several countries ${ }^{10}$.

For antivirals to significantly reduce transmission, prophylactic use is necessary. Large-scale prophylaxis has the potential to limit spread substantially in a developed country ${ }^{11}$, but the very large stocks of drug necessary make this policy impractical if the pandemic is already global. However, might such a policy nonetheless be a feasible strategy if applied at the source of a new pandemic, when repeated human-tohuman transmission is first observed? Here we address this question, and focus on identifying the threshold level of transmissibility below which containment of any new pandemic strain might be feasible.

\section{Modelling pandemic spread}

We modelled pandemic spread in Southeast Asia, as this region remains the focus of the ongoing avian $\mathrm{H} 5 \mathrm{~N} 1$ epidemic and is where most human cases have occurred. Data availability led us to model Thailand rather than any perceived greater risk of emergence compared to other countries in the region; however, we believe our conclusions are also valid for other parts of Southeast Asia.

We constructed a spatially explicit simulation of the 85 million people residing in Thailand and in a $100-\mathrm{km}$ wide zone of contiguous neighbouring countries. The model explicitly incorporates households, schools and workplaces, as these are known to be the primary contexts of influenza transmission ${ }^{12-14}$ (see Fig. 1 and Methods) and because control measures can readily target these locations. Random contacts in the community associated with day-to-day movement and travel were also modelled.

\section{Natural history and transmission parameters}

Fundamental to the feasibility of any containment strategy is being able to quantify the transmissibility of the emergent virus, $R_{0}$. Reliable past estimates of transmissibility are rare, perhaps owing to the antigenic diversity of influenza and the consequent complex effect of population immunity on transmission.

We re-analysed incubation period and household transmission data for human influenza (see Methods) and derived new natural history parameters, which predict a profile of infectiousness over time that is remarkably consistent with viral shedding data from experimental infection studies (see Fig. $1 \mathrm{~g}$ and ref. 15). This profile

${ }^{1}$ Department of Infectious Disease Epidemiology, Faculty of Medicine, Imperial College London, St Mary's Campus, Norfolk Place, London W2 1PG, UK. ${ }^{2}$ The Institute of Mathematical Sciences, Imperial College London, 53 Princes Gate, London, SW7 2PG, UK. ${ }^{3}$ Department of International Health, Johns Hopkins Bloomberg School of Public Health, 615 North Wolfe Street, Baltimore, Maryland 21205, USA. ${ }^{4}$ INSERM U707, 27 rue Chaligny, Paris 75571, cedex 12, France. ${ }^{5}$ Department of Community Medicine, 5/F William M. W. Mong Block, Faculty of Medicine Building, The University of Hong Kong, 21 Sassoon Road, Hong Kong. ${ }^{6}$ Bureau of Epidemiology, Department of Diseases Control, Ministry of Public Health, Tivanonda Road, Nonthaburi 11000, Thailand. 
gives an estimate of the serial interval or generation time, $T_{\mathrm{g}}$ (the average interval from infection of one individual to when their contacts are infected), of 2.6 days, compared with the value of $\sim 4$ days assumed by most previous modelling studies ${ }^{16}$. Re-analysis of both US and UK 1918 pandemic mortality data using this new value of $T_{\mathrm{g}}$ revises pandemic influenza $R_{0}$ estimates ${ }^{5}$ downwards to approximately 1.8 (Fig. If and Supplementary Information). This yields a predicted infection attack rate of 50-60\% during a pandemic, consistent with what was seen in the first and second waves of past pandemics (see Supplementary Information). An $R_{0}$ value of 1.8 is also consistent with annual interpandemic attack rates seen in households where all members were highly susceptible to the prevalent $\operatorname{strain}^{17}$ (see Supplementary Information). We also assumed that $50 \%$ of infections result in clinically recognizable symptoms, with the other $50 \%$ being too mild to be diagnosed clinically ${ }^{18}$.

We cannot be certain that these parameter estimates would be applicable to any new pandemic strain. It is possible that the mutations or reassortment events that give rise to the new viral strain might initially increase its transmissibility only slightly over the $R_{0}=1$ threshold for self-sustaining transmission. In that case, additional mutations would have to accumulate for viral fitness to increase to its maximum. Given the extended viral shedding (and symptomatic disease) seen in severe human cases of avian $\mathrm{H} 5 \mathrm{~N} 1$ infection, this might also mean that the $T_{\mathrm{g}}$ of the initial pandemic strain could be considerably greater than for currently circulating human influenza viruses. We therefore examine the ability of control measures to contain pandemic spread not just at a single value of $R_{0}$, but for different values in the range $1<R_{0}<2$, and analyse model sensitivity to the assumed value of $T_{\mathrm{g}}$.

\section{Baseline epidemic dynamics}

We consider the scenario that a new transmissible $\left(R_{0}>1\right)$ pandemic strain arises as a result of mutations or a reassortment event in a single individual infected with an avian virus. We seed simulations with a single infection in the most rural third of the population (that is, with the lowest population density), assuming that rural populations are most likely to be exposed to the avian virus. Figure 2 shows the typical pattern of spread for an emergent pandemic initiated by such a seeding event assuming $R_{0}=1.5$, but note that for low $R_{0}$, most epidemics seeded by a single individual go extinct by chance before becoming established in the population.

The pattern of spatial spread (Fig. 2a and Supplementary Video 1) is of interest: for the first 30 days, cases tend to be limited to the region around the seeding location, with few 'sparks' outside that area. However, as case numbers increase exponentially, so does the frequency with which infection events span large distances, and the epidemic rapidly transforms from being predominantly local to country-wide between days 60 and 90 (Fig. 2a-c). Any containment policy needs to be effective before this transition, in part because logistical constraints are likely to preclude containment of a widely disseminated epidemic, but also because the probability of international export of infection becomes high once case numbers reach the thousands (Hollingsworth, D., N. M. F. \& Anderson, R. M., unpublished observations).

For $R_{0}=1.5$, the epidemic in the modelled population of 85 million peaks around day 150 and is largely over by day 200 (Fig. 2b), at which point $33 \%$ of the population has been infected (Fig. 2d). At $R_{0}=1.8$, the epidemic peaks around day 100 and infects about $50 \%$ of the population.
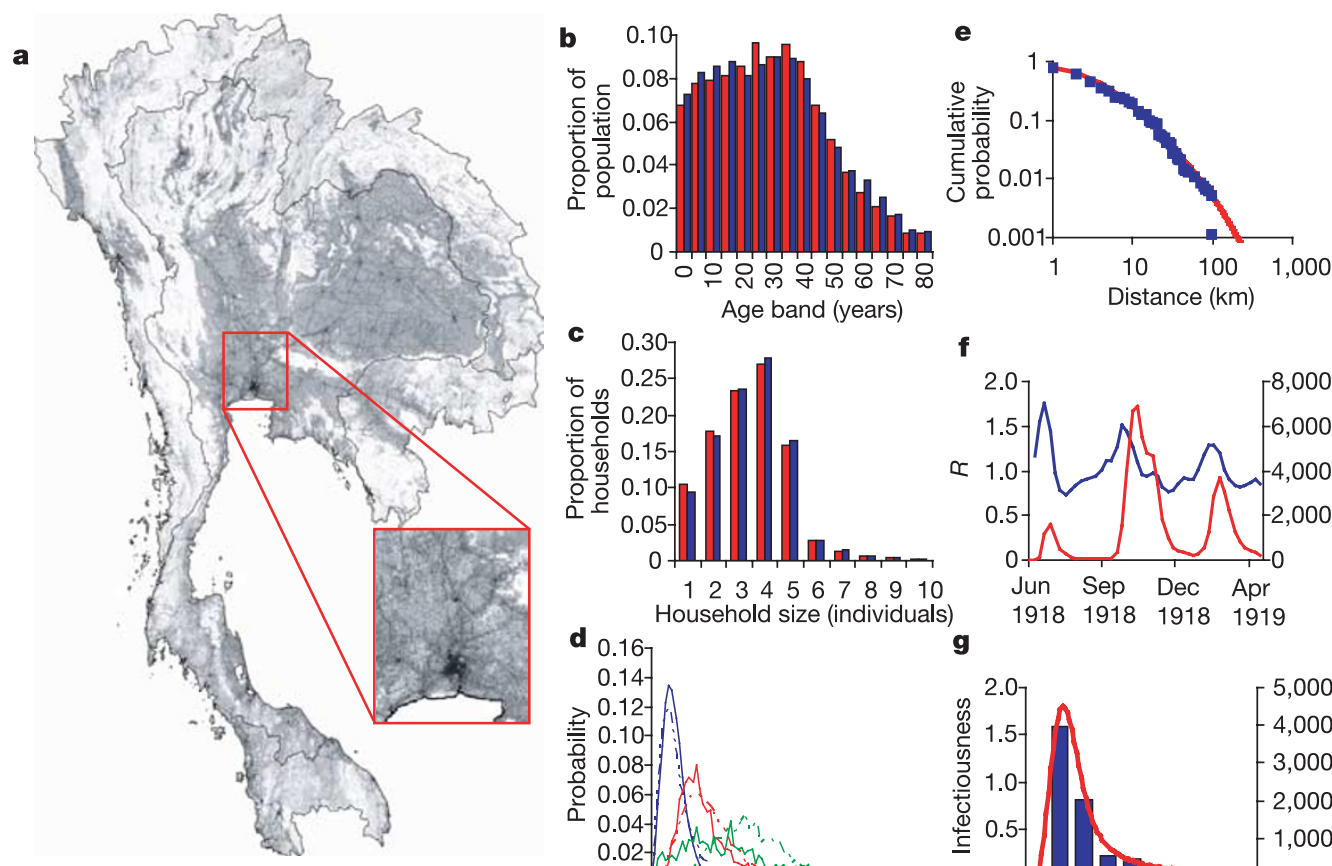

f
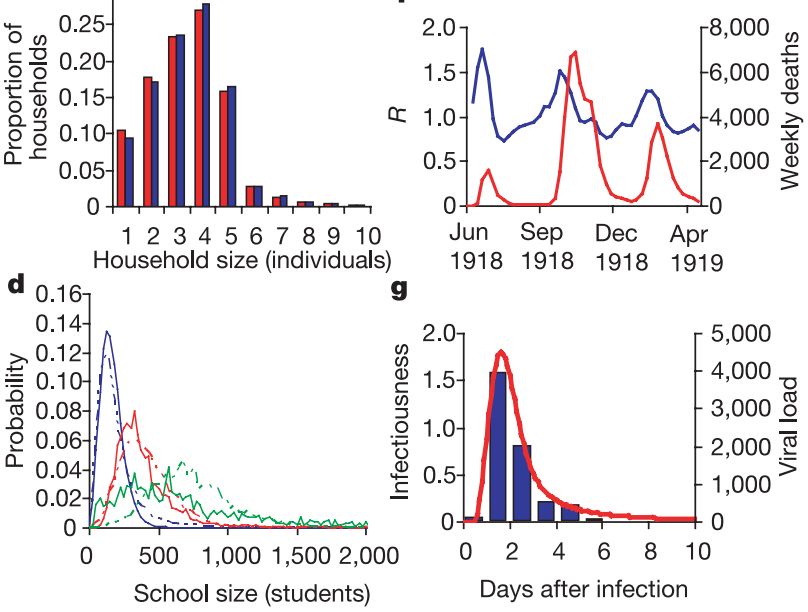

Figure 1 | Data. a, Modelled population density of Thailand and 100-km contiguous zone of neighbouring countries, based on Landscan ${ }^{24}$ data and plotted on a logarithmic scale (light for low density, dark for high density). Inset shows Bangkok in more detail. $\mathbf{b}$, Age distribution of Thai population in 2003 in 5-yr bands (blue), and the corresponding age distribution of the simulated population (red). c, As b but showing distribution of household sizes. d, Observed (solid lines) and modelled (dashed lines) distributions of school sizes (blue, elementary; green, secondary; red, mixed). e, Probability of travelling over a certain distance to work, estimated from data (blue) and from the simulated population (red). $\mathbf{f}$, Weekly excess influenza-related mortality in 1918-1919 in Great Britain (red), and corresponding estimates of the reproduction number $R$ (blue), calculated assuming $T_{\mathrm{g}}=2.6 . \mathrm{g}$, Viral shedding data for experimental influenza infection ${ }^{15}$ (expressed in tissue culture infective doses $\left(\mathrm{TCID}_{50}\right.$ ) per $\mathrm{ml}$ of nasal lavage fluid) compared with the modelled profile of infectiousness over time. Note that the infectiousness profile was not fitted to shedding data. See Methods and Supplementary Information for more details. 


\section{Effect of antiviral prophylaxis}

In evaluating containment strategies, we focus on two principal outcome measures: (1) the probability of preventing a large outbreak (which would eventually lead to a global pandemic), and (2) the number of courses of drug (assumed here to be oseltamivir) required to achieve containment.

Blanket prophylaxis of an entire country or region should be able to eliminate a pandemic virus with an $R_{0}$ of 3.6 or greater (see Methods). However, such a policy would require enough drug to prophylax everyone for up to three weeks (that is, at least two courses per person) and is hence unfeasible. Targeted strategies are therefore needed to minimize drug usage while maximizing effect.

Social targeting is the most straightforward approach. This involves prophylaxing individuals in the same household, school or workplace as a newly diagnosed symptomatic case. Unfortunately, if such a policy is only initiated after 20 or more cases, purely social targeting only has a $\geq 90 \%$ probability of eliminating the pandemic strain if $R_{0} \leq 1.25$ (lowest curve of Fig. 3a; see also Supplementary Information). In reality, at least ten cases might have to be detected to be sure that viral transmissibility had significantly increased ${ }^{19}$, and detection and decision-making delays could easily mean 20-30 cases had arisen before policy initiation. A containment policy will therefore probably have to go beyond social targeting in order to succeed. As most community contacts are local, geographic targetingnamely, prophylaxing the whole population in the neighbourhood of the household in which a case is detected-is an obvious policy extension, but one that will no doubt greatly increase the logistical challenges to delivery. In the absence of detailed administrative boundary data, we simulated geographic targeting as the prophylaxis of the population within a ring of a certain radius centred around each detected case, but in practice targeting administrative areas is likely to be more practical. For social or geographic prophylaxis, we assume that individuals are given a single course of ten days of drug, after which time they come off the drug unless more cases have arisen in their vicinity, in which case a second round of prophylaxis is delivered. The policies therefore cease automatically within ten days of the last case being reported.
Our analysis indicates that the additional effort required to deliver a geographic policy pays substantial dividends in terms of policy effectiveness. With a two-day delay from case onset to prophylaxis, a $5-\mathrm{km}$ ring policy is able to contain pandemics with an $R_{0}$ of 1.5 (Fig. 3a) at the cost of an average of 2 million courses (Fig. 3b), but the maximum number of courses needed can increase by an (unfeasible) order of magnitude for scenarios in which cases arise in Bangkok at an early stage of the outbreak. Policy effectiveness increases with the radius of the treatment ring selected (but little benefit is gained from exceeding $10 \mathrm{~km}$ ), as does the number of courses required (Fig. 3b). Policy outcome is still sensitive to the speed of case detection and drug delivery, but containment is always substantially better than for the purely socially targeted policy (Fig. 3d).

As pure radial prophylaxis is costly in terms of drug, we also examined a policy variant that limits the number of people targeted for prophylaxis per case by only targeting the nearest $m$ people (where $m=10,000-50,000)$ within $10 \mathrm{~km}$ of a newly diagnosed case. In areas of low population density, this drug-sparing policy has the same effect as a pure $10-\mathrm{km}$ ring policy, but in high-density areas many fewer courses of drug are used. The improved effectiveness in rural areas outweighs decreased effectiveness in urban areas, resulting in a greater effect than a pure 5-km ring policy and much lower drug use (Fig. 3e, f).

Epidemiologically, elimination occurs either because the treatment strategy reduces $R_{0}$ to below 1 , or because it reduces $R_{0}$ to close to 1 when the epidemic is small, thereby enhancing the probability of random extinction. For scenarios in which the pandemic strain is successfully eliminated, geographic spread is usually limited. For example, the root mean square (r.m.s.) radius of spread is $27 \mathrm{~km}$ for $R_{0}=1.5$ using the $5-\mathrm{km}$ radial geographic targeting strategy. When containment is successful, total case numbers are also limited to an average of fewer than 150 cases.

\section{Policies to increase social distance}

Measures to increase social distance have been used in past pandemics and remain important options for responding to future pandemics ${ }^{1}$. However, predicting the effect of policies such as closing schools and workplaces is difficult, as potentially infectious contacts

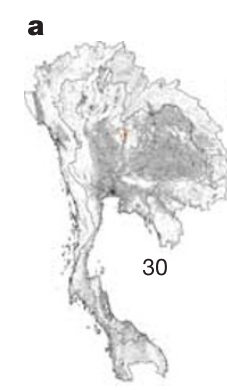

b
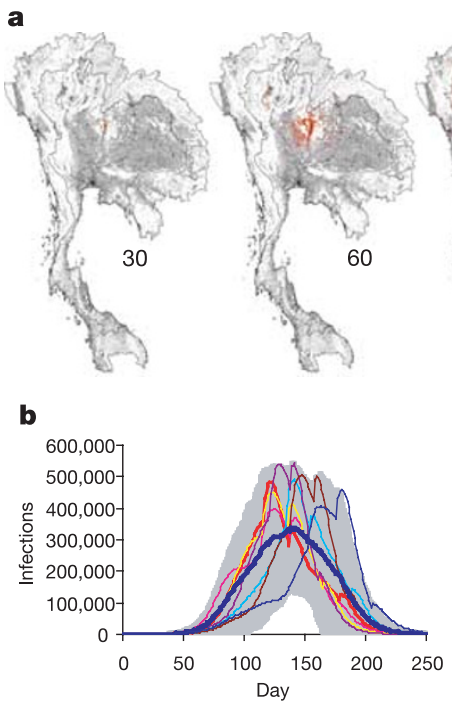

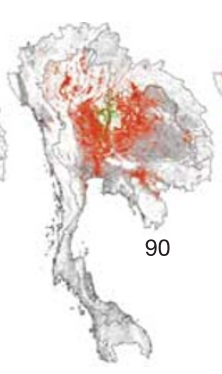

c

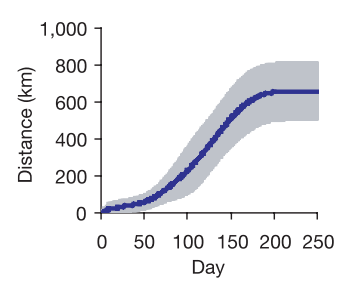

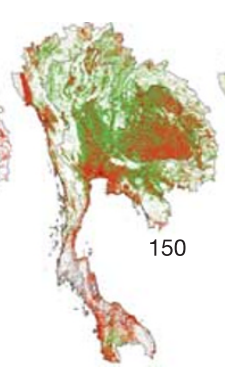

d

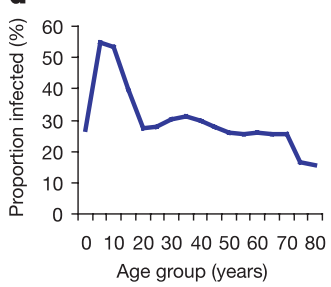

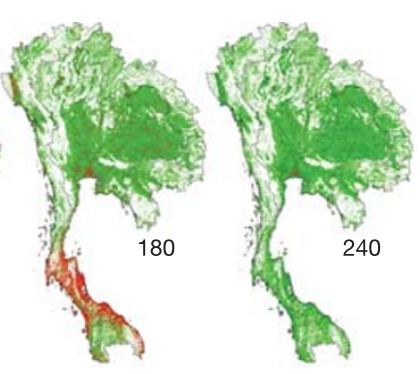

e

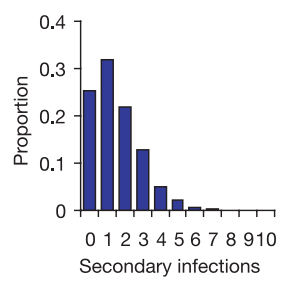

Figure 2 | Expected pattern of spread of an uncontrolled epidemic. a, Time sequence (in days) of an epidemic, showing spreading in a single simulation of an epidemic with $R_{0}=1.5$. Red indicates presence of infected individuals, green the density of people who have recovered from infection or died. b, Daily incidence of infection over time for $R_{0}=1.5$ in the absence of control measures. Thick blue line represents average for realizations resulting in a large epidemic, grey shading represents $95 \%$ confidence limits of the incidence time series. Multiple coloured thin lines show a sample of realizations, illustrating a large degree of stochastic variability. c, Root mean square (r.m.s.) distance from the seed infective for individuals infected since the start of the epidemic as a function of time. Thick blue line represents average distance for realizations resulting in a large epidemic, grey shading represents $95 \%$ limits. d, Proportion of the population infected by age for $R_{0}=1.5$, averaged across realizations that result in large epidemics. The infection attack rate is $33 \%$ for $R_{0}=1.5$ and $50 \%$ for $R_{0}=1.8$. e, Distribution of the number of secondary cases per primary case during the exponential growth phase of a $R_{0}=1.5$ epidemic. Between 50 and 1,000 realizations were used to calculate all averages (see Supplementary Information). 
may be displaced into other settings. Furthermore, it is likely that population contact rates change spontaneously (as well as a result of policy) during severe epidemics (for example, 1918) in response to the perceived risk. Therefore, the estimates of pandemic transmissibility we derive from past pandemics might implicitly incorporate the effects of some degree of social distancing.

We are therefore deliberately conservative in the assumptions made here regarding the effect of school and workplace closure, by assuming that household and random contact rates increase by $100 \%$ and $50 \%$, respectively, for individuals no longer able to attend school or work. Figure 4a illustrates how adding area-based school and workplace closure to a drug-sparing prophylaxis policy increases policy effectiveness significantly, with the combined policy having a $>90 \%$ chance of elimination for $R_{0}=1.7$.

Quarantine zones, in which movements in and out of the affected area are restricted, are another strategy for enhancing containment, and may in any case be thought necessary to prevent population flight from affected areas or deliberate entrance of people into prophylaxis zones to receive drug. Figure $4 \mathrm{a}$ (see also Supplementary Video 2) shows that such an area quarantine strategy can greatly increase the effectiveness (to $90 \%$ containment at $R_{0}=1.8$ ) of radial geographic targeted prophylaxis even if only $80 \%$ effective at reducing movements. Combining school and workplace closure with area quarantine and prophylaxis further increases policy effectiveness (90\% containment at $R_{0}=1.9$ ), and equally importantly, increases the robustness of the policy to shortcomings in case identification or
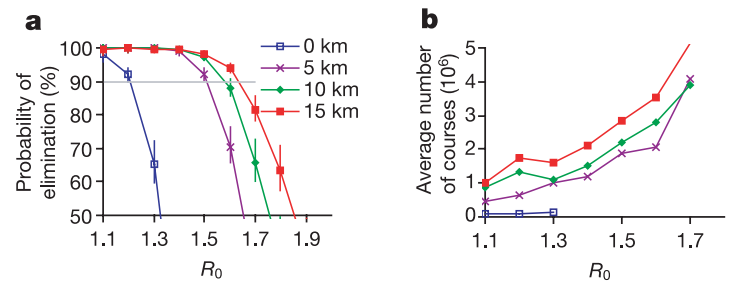

c
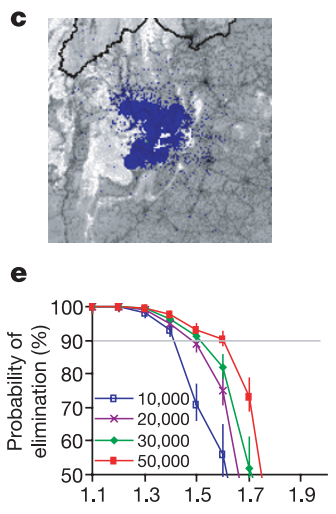

$R_{0}$

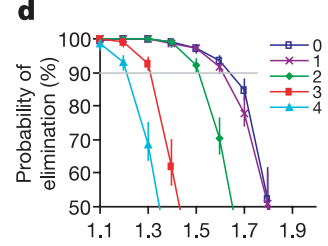

$\mathbf{f}$

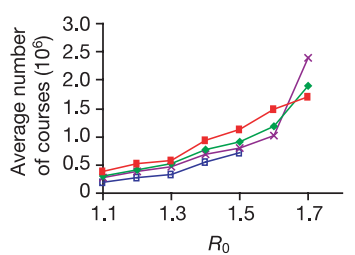

Figure 3 | Prophylaxis strategies. We assume $90 \%$ of clinical cases (45\% of infections) are detected. Social targeting assumes prophylaxis of $90 \%$ of household members and $90 \%$ of pupils or colleagues in $90 \%$ of the schools or workplaces with detected cases. Geographic targeting assumes $90 \%$ of people within 5,10 or $15 \mathrm{~km}$ of a detected case are also prophylaxed. a, Probability of eliminating an otherwise large epidemic using social and geographic targeting, as a function of $R_{0}$ of the new strain and the radius of prophylaxis. Results assume policy initiation after detection of 20 cases and a two-day delay from case detection to prophylaxis. Error bars show exact $95 \%$ confidence limits. $\mathbf{b}$, Same as $\mathbf{a}$, but showing average number of drug courses required for containment of an otherwise large outbreak. c, Map of northern Thailand $(150 \times 150 \mathrm{~km}$ square $)$, showing the extent of spread during one contained $R_{0}=1.8$ epidemic assuming $10-\mathrm{km}$ radial prophylaxis and other parameters as in $\mathbf{a}$. Treated areas shown in blue. d, Same as a, but varying the delay (0-4 days) from case detection to prophylaxis for the 5-km radius policy. e, f, Same as $\mathbf{a}$ and $\mathbf{b}$, but for drugsparing policies that target only the nearest $10,000-50,000$ people within $10 \mathrm{~km}$ of a detected case. Error bars show exact $95 \%$ confidence limits. treatment rates. For all these policies, containment is typically achieved after fewer then 200 cases have been detected.

\section{Logistical constraints and sensitivity to parameter assumptions}

Other constraints may affect the ability of public health authorities to deliver containment policies. Figure $4 \mathrm{c}$ shows that the size of an antiviral stockpile can have a substantial effect on policies that use pure radial geographic prophylaxis, as very large numbers of courses are required to prophylax populations around cases arising in large urban areas. However, policies using drug-sparing, geographically targeted prophylaxis (Fig. 4d) retain high effectiveness provided that at least 3 million drug courses are available. For scenarios in which containment fails with a finite stockpile, Fig. 4e shows that even an unsuccessful containment strategy can delay widescale spread by a month or more-a potentially critical window of opportunity for accelerating vaccine production.

Another possible constraint is that capacity to implement these containment policies might not be present in all countries in the region. A policy restricted to one country alone might have a substantially reduced chance of success (Fig. $4 \mathrm{f}$ and Supplementary Video 3 ) should the initial case cluster arise in a border region.

Multiple assumptions are inevitably made when undertaking preparedness modelling for a future emergent infection. Sensitivity analyses are therefore critical for assessing the robustness of policy conclusions. Here, critical assumptions not already discussed include (1) the ratio of within-place to community transmission, (2) the
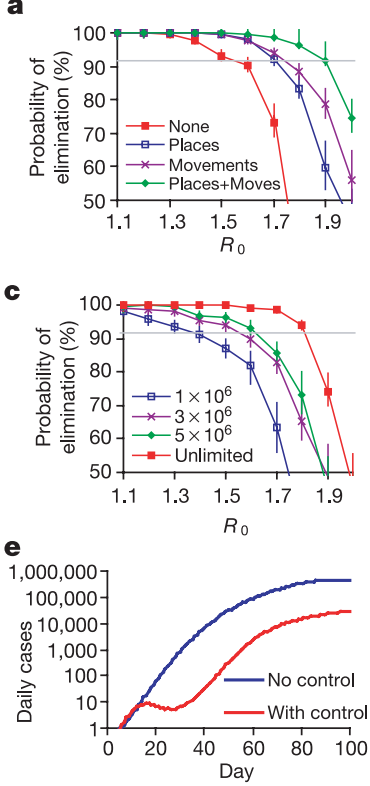

b

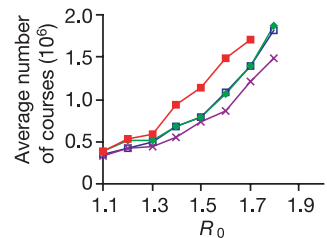

d
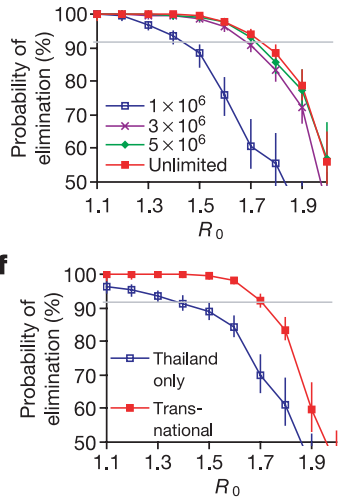

Figure 4 | Social distance measures. a, b, Same as Fig. 3a, b, but showing the effect of drug-sparing prophylaxis (50,000 courses per case, as Fig. 3e) together with: no social distance measures (red; as Fig. 3); 21-day closure of $90 \%$ of schools and $50 \%$ of workplaces within $5 \mathrm{~km}$ of a detected case (blue); $80 \%$ 'area quarantine' (that is, $80 \%$ reduction of movement in and out of a zone defined by merging $5-\mathrm{km}$ rings around all detected cases) for 21 days (magenta); or a combination of school/workplace closure and area quarantine (green). c, Same as a but showing the effect of limiting availability of antiviral drugs to 1, 3 or 5 million courses on the effectiveness of the combined area quarantine and $5-\mathrm{km}$ radial prophylaxis policy. d, Same as c but for drug-sparing geographic prophylaxis (50,000 courses per case) plus area quarantine. e, Case incidence over time without pandemic control measures and with the 3 million course policy of $\mathbf{d}$, showing the approximate one-month delay achieved even when containment is unsuccessful $\left(R_{0}=1.9\right)$. $\mathrm{f}$, Same as a but showing the reduction in policy effectiveness seen if the combined school/workplace closure and drug-sparing prophylaxis policy is restricted to Thailand alone. Error bars show exact $95 \%$ confidence limits. 
expected generation time, $T_{\mathrm{g}}$, of a new pandemic strain (largely determined by the duration of viral shedding and therefore infectiousness), (3) the level of heterogeneity in individual infectiousness (for example, 'superspreaders ${ }^{20}$ ), (4) antiviral efficacy/take-up, and (5) the sensitivity and specificity of case detection during the control programme. The effect of these assumptions on model output is presented in the Supplementary Information. In summary, points (4) and (5) are the most critical, as one might expect. If antiviral coverage or efficacy is considerably less than assumed, then policy effectiveness is substantially reduced. Similarly, if surveillance picks up fewer than $40 \%$ of infections (that is, $80 \%$ of symptomatic cases), policy effectiveness is again reduced. Poor surveillance specificity (that is, false positives) has an indirect effect on effectiveness as a result of wasted drug and logistical capacity.

\section{Conclusions}

We have shown that containment and elimination of an emergent pandemic strain of influenza at the point of origin is feasible using a combination of antiviral prophylaxis and social distance measures. A key conclusion is the need for multiple approaches: simple socially targeted prophylaxis is unlikely to be sufficient if the emergent virus has transmissibility levels near those of previous pandemic viruses. Geographically targeted policies are needed to achieve high levels of containment, with area quarantine being particularly effective at boosting policy effectiveness. The only scenario under which purely socially targeted strategies might be sufficient would be if viral transmissibility evolved incrementally and the emergent virus initially had an $R_{0}$ only slightly above 1 (see Supplementary Information); however, $R_{0}$ will be probably be uncertain at the time at which containment policies have to be implemented, arguing for precautionary policies that assume transmissibility comparable with that of past pandemics.

A number of key criteria must be met for a high probability of success: (1) rapid identification of the original case cluster, (2) rapid, sensitive case detection and delivery of treatment to targeted groups, preferably within $48 \mathrm{~h}$ of a case arising, (3) effective delivery of treatment to a high proportion of the targeted population, preferably $>90 \%$, (4) sufficient stockpiles of drug, preferably 3 million or more courses of oseltamivir, (5) population cooperation with the containment strategy and, in particular, any social distance measures introduced, (6) international cooperation in policy development, epidemic surveillance and control strategy implementation. Containment is unlikely if $R_{0}$ exceeds 1.8 for the new pandemic strain. Although our analysis of past pandemics suggests that transmissibility will fall below this threshold, it is unlikely that sufficient data will exist to verify this before a containment policy has to be introduced.

The mathematical model we have used to examine the feasibility of pandemic containment is perhaps the largest-scale detailed epidemic micro-simulation yet developed. A key goal of the modelling was parsimony. Although the representation of the population is detailed, this detail is underpinned by available demographic data. The natural history parameters used here have been estimated from primary data on existing influenza strains. The model has five key transmission parameters, of which two were estimated from household data and the remaining three were qualitatively calibrated to historical age-dependent attack rates. We believe that this type of simulation will increasingly become a standard tool for preparedness planning and modelling of new disease outbreaks.

Given the set of criteria listed above for successful containment, the obstacles to practical implementation of such a strategy are undoubtedly formidable. Surveillance is perhaps the single greatest challenge. Success depends on early identification of the first cluster of cases caused by the pandemic strain ${ }^{19}$, and on detection of a high proportion of ongoing cases. Some level of mildly symptomatic infection is to be expected (and has been observed for human H5N1 infections ${ }^{21}$ ), but key to successful containment is the proportion of such cases and their infectiousness. Should the high pathogenicity of recently reported human infections with the H5N1 virus be even partly maintained, then containment might paradoxically be more likely, as case-ascertainment levels would be higher.

Achieving the rapid delivery of antiviral drugs to a large proportion of the population raises many challenges. Thailand, the country modelled here, is one of the best-prepared and equipped countries in the region in terms of being able to implement a large-scale and very rapid public health intervention. Other countries need considerable development in basic healthcare and disease surveillance infrastructure in order to meet the needs of containment.

Antiviral resistance represents a currently unquantifiable challenge to a prophylaxis-based containment strategy. The key will not be whether genotypic or clinical resistance is seen in a percentage of individuals, but whether resistant viruses are capable of self-sustaining transmission (that is, have $R_{0}>1$ ). Current evidence indicates that fitness deficits in oseltamivir-resistant strains mean that their transmissibility is limited ${ }^{22,23}$, but we cannot rule out the possibility that compensatory mutations that increase transmissibility might be selected. If a transmissible resistant strain did emerge during implementation of a containment policy, it would be essential for prophylaxis to cease, lest the wild-type virus be eliminated and the world be left with a pandemic of resistant virus. If prophylaxis were abandoned, the likely higher fitness of the wild-type virus would give every chance for the resistant strain to become excluded from the population.

A feasible strategy for containment of the next influenza pandemic offers the potential to prevent millions of deaths. It is therefore in the interest of all countries to contribute to ensuring that resources, infrastructure and collaborative relationships are in place within the region most likely to be the source of a new pandemic. The challenges are great, but the costs of failure are potentially so catastrophic that it is imperative for the international community to prepare now, to ensure that containment is given the best possible chance of success.

\section{METHODS}

Demographic data. The model used Landscan data ${ }^{24}$ to generate a simulated population realistically distributed across geographic space (Fig. 1a). Thai census data $^{25,26}$ on household size and age distributions were used for demographic parameterization (Fig. 1b, c). Data from the Thai National Statistical Office ${ }^{26}$ were used to determine the number and proportions of children in school as a function of age, and data from the Thai Department of Education on 24,000 schools (available from the authors upon request) were used to determine the distribution of school sizes (Fig. 1d). Data on travel distances within Thailand were limited; here we used data collected in the 1994 National Migration Survey ${ }^{27,28}$ on distances travelled to work (Fig. 1e and Supplementary Information) to estimate movement kernel parameters. The best-fit kernel had asymptotic power-law form as a function of distance $d$ given by $f(d) \sim$ $1 /\left[1+(d / a)^{b}\right]$, where $a=4 \mathrm{~km}$ and $b=3.8$. Thai workplace sizes ${ }^{29}$ also follow a power-law distribution ${ }^{30}$, with an estimated maximum single workplace size of approximately 2,300 and a mean of 21 individuals.

Disease data. The natural history of any H5-based pandemic strain will not be known until it emerges, so we used parameter estimates for current human influenza subtypes, and used sensitivity analyses to investigate what effect deviation from these estimates would have on policy effectiveness (see Supplementary Information). The mean \pm s.d. of the incubation period distribution was estimated as $1.48 \pm 0.47$ days, on the basis of data from a multipleexposure event occurring on an aeroplane ${ }^{31}$.

We adopt a more biologically realistic approach than most previous modelling studies (but see ref. 32), and rather than assuming that infectiousness is constant from the end of the latent period until recovery, we model it as a function, $\kappa(T)$ (assumed normalized), depending on the time elapsed from the end of the latent period. The generation time, $T_{\mathrm{g}}$, is given by the mean latent period plus $\int_{0}^{\infty} T \kappa(T) \mathrm{d} T$. Experimental infection data ${ }^{33}$ indicate the start of symptoms to be coincident with a sharp increase in viral shedding, so we assume that infectiousness starts at the end of the incubation period. We further assume a 0.25-day delay from when symptoms start to when diagnosis or healthcareseeking behaviour is likely. We used bayesian methods (see Supplementary Information) to estimate $\kappa(T)$ from data collected in a recent household study of respiratory disease incidence ${ }^{34,35}$. Combined with the estimated incubation period distribution, this gives the profile of infectiousness shown in Fig. 1g. 
$T_{\mathrm{g}}$ is estimated as 2.6 days ( $95 \%$ credible interval: $2.1-3.0$ ), which is shorter than previously assumed (but see ref. 36).

Transmission model. The model is a stochastic, spatially structured, individualbased discrete time simulation. Individuals are co-located in households, with households being constructed to reflect typical generational structure while matching empirical distributions of age structure and household size for Thailand (Fig. 1b, c). Households are randomly distributed in the modelled geographic region, with a local density determined by the Landscan data ${ }^{24}$. In any time-step of $\Delta T=0.25$ days, a susceptible individual $i$ has probability $1-\exp \left(-\lambda_{i} \Delta T\right)$ of being infected, where $\lambda_{i}$ is the instantaneous infection risk for individual $i$. Infection risk comes from 3 sources: (1) household, (2) place, and (3) random contacts in the community. The last of these depends on distance, representing random contacts associated with movements and travel, and is the only means by which infection can cross national borders. Analysis of household infection data (see Supplementary Information), gave a withinhousehold $R_{0}$ of 0.6 and an overall $R_{0}$ of 1.8. We partition non-household transmission to give levels of within-place transmission comparable with household transmission (that is, $R_{0} \approx 0.6$ ) and to qualitatively match 1957 influenza pandemic age-specific attack rates. When varying $R_{0}$, the relative proportions of household, place and community transmission were kept fixed. Full model details are given in the Supplementary Information.

Antiviral drug action. We use recent statistically rigorous estimates of antiviral efficacy $^{37}$, but these are broadly consistent with previous estimates ${ }^{22}$. Prophylaxis of uninfected individuals is assumed to reduce susceptibility to infection by $30 \%$, reduce infectiousness if infection occurs by $60 \%$, and reduce the probability of clinically recognizable symptoms by $65 \%$ (ref. 37 ). In theory, blanket prophylaxis of a population should be able to contain a pandemic with an $R_{0}$ of $1 /[(1-0.6)(1-0.3)]$, or approximately 3.6. Treatment of a symptomatic case is assumed to reduce infectiousness by $60 \%$ from when treatment is initiated. Overall, for the parameter values used here, antiviral treatment of a symptomatic case can reduce total infectiousness throughout the course of infection by a maximum of $28 \%$.

\section{Received 2 June; accepted 14 July 2005.}

\section{Published online 3 August 2005}

1. World Health Organisation. Avian influenza: assessing the pandemic threat 〈http://www.who.int/csr/disease/influenza/WHO_CDS_2005_29/en/ index.html $\rangle$ (2005)

2. Abbott, A. \& Pearson, H. Fear of human pandemic grows as bird flu sweeps through Asia. Nature 427, 472-473 (2004)

3. Spicer, C. C. \& Lawrence, C. J. Epidemic influenza in Greater London. J. Hyg. (Lond.) 93, 105-112 (1984).

4. Fleming, D. M., Zambon, M., Bartelds, A. I. M. \& de Jong, J. C. The duration and magnitude of influenza epidemics: A study of surveillance data from sentinel general practices in England, Wales and the Netherlands. Eur. J. Epidemiol. 15, 467-473 (1999)

5. Mills, C. E., Robins, J. M. \& Lipsitch, M. Transmissibility of 1918 pandemic influenza. Nature 432, 904-906 (2004).

6. Anderson, R. M. \& May, R. M. Infectious Diseases of Humans: Dynamics and Control (Oxford Univ. Press, Oxford, 1992).

7. Stohr, K. \& Esveld, M. Will vaccines be available for the next influenza pandemic? Science 306, 2195-2196 (2004)

8. Leneva, I. A., Roberts, N., Govorkova, E. A., Goloubeva, O. G. \& Webster, R. G. The neuraminidase inhibitor GS4104 (oseltamivir phosphate) is efficacious against A/Hong Kong/156/97 (H5N1) and A/Hong Kong/1074/99 (H9N2) influenza viruses. Antiviral Res. 48, 101-115 (2000)

9. Brooks, M. J., Sasadeusz, J. J. \& Tannock, G. A. Antiviral chemotherapeutic agents against respiratory viruses: where are we now and what's in the pipeline? Curr. Opin. Pulm. Med. 10, 197-203 (2004).

10. Coombes, R. UK stocks up on antiviral drug to tackle flu outbreak. Br. Med. J. 330, 495 (2005)

11. Longini, I. M. Jr, Halloran, M. E., Nizam, A. \& Yang, Y. Containing pandemic influenza with antiviral agents. Am. J. Epidemiol. 159, 623-633 (2004).

12. Viboud, C. et al. Risk factors of influenza transmission in households. Br. J. Gen. Pract. 54, 684-689 (2004)

13. Principi, N., Esposito, S., Gasparini, R., Marchisio, P. \& Crovari, P. Burden of influenza in healthy children and their households. Arch. Dis. Child. 89, 1002-1007 (2004)

14. Heymann, A., Chodick, G., Reichman, B., Kokia, E. \& Laufer, J. Influence of school closure on the incidence of viral respiratory diseases among children and on health care utilization. Pediatr. Infect. Dis. J. 23, 675-677 (2004)

15. Hayden, F. G. et al. Local and systemic cytokine responses during experimental human influenza A virus infection. Relation to symptom formation and host defense. J. Clin. Invest. 101, 643-649 (1998).

16. Elveback, L. R. et al. An influenza simulation model for immunization studies Am. J. Epidemiol. 103, 152-165 (1976).

17. Longini, I. M. Jr, Koopman, J. S., Haber, M. \& Cotsonis, G. A. Statistical inference for infectious diseases. Risk-specific household and community transmission parameters. Am. J. Epidemiol. 128, 845-859 (1988).

18. Fox, J. P., Hall, C. E., Cooney, M. K \& Foy, H. M. Influenzavirus infections in Seattle families, 1975-1979. I. Study design, methods and the occurrence of infections by time and age. Am. J. Epidemiol. 116, 212-227 (1982).

19. Ferguson, N. M., Fraser, C., Donnelly, C. A., Ghani, A. C.\& Anderson, R. M. Public health risk from the avian H5N1 influenza epidemic. Science 304, 968-969 (2004)

20. Riley, S. et al. Transmission dynamics of the etiological agent of SARS in Hong Kong: Impact of public health interventions. Science 300, 1961-1966 (2003).

21. World Health Organisation. WHO inter-country consultation influenza A/ H5N1 in humans in Asia. http://www.who.int/csr/disease/avian_influenza/ H5N1\%20Intercountry\%20Assessment\%20final.pdf (2005).

22. Ferguson, N. M., Mallett, S., Jackson, H., Roberts, N. \& Ward, P. A populationdynamic model for evaluating the potential spread of drug-resistant influenza virus infections during community-based use of antivirals. J. Antimicrob. Chemother. 51, 977-990 (2003).

23. Herlocher, M. L. et al. Influenza viruses resistant to the antiviral drug oseltamivir: transmission studies in ferrets. J. Infect. Dis. 190, 1627-1630 (2004).

24. Oakridge National Laboratory. Landscan global population data. http:// www.ornl.gov/sci/gist/landscan (2003).

25. National Statistical Office Thailand. Population Census 2000. http:// web.nso.go.th/pop2000/pop_e2000.htm (2000).

26. National Statistical Office Thailand. Population and Labor Statistics. http:// web.nso.go.th/eng/stat/subject/subject.htm\#cata1 (1999).

27. National Statistical Office Thailand. National Migration Survey. http:// web.nso.go.th/eng/stat/migrant/migrant.htm and http://opr.princeton.edu/ archive/nmst/ (1994)

28. Chamratrithirong, A. et al. National Migration Survey of Thailand (Institute for Population and Social Research, Mahidol Univ., Bangkok, 1995).

29. Ministry of Labour Thailand. Yearbook of Labour Statistics. http:// www1.mol.go.th/download/chap031-2.pdf (2003).

30. Axtell, R. L. Zipf distribution of U.S. firm sizes. Science 293, 1818-1820 (2001).

31. Moser, M. R. et al. An outbreak of influenza aboard a commercial airliner. Am. J. Epidemiol. 110, 1-6 (1979).

32. Fraser, C., Riley, S., Anderson, R. M. \& Ferguson, N. M. Factors that make an infectious disease outbreak controllable. Proc. Natl Acad. Sci. USA 101, 6146-6151 (2004)

33. Hayden, F. G. et al. Use of the oral neuraminidase inhibitor Oseltamivir in experimental human influenza. J. Am. Med. Assoc. 282, 1240-1246 (1999).

34. Carrat, F. et al. Influenza burden of illness: estimates from a national prospective survey of household contacts in France. Arch. Intern. Med. 162, 1842-1848 (2002)

35. Cauchemez, S., Carrat, F., Viboud, C., Valleron, A. J. \& Boelle, P. Y. A Bayesian MCMC approach to study transmission of influenza: application to household longitudinal data. Stat. Med. 23, 3469-3487 (2004).

36. Flahault, A. et al. Modelling the 1985 influenza epidemic in France. Stat. Med. 7 1147-1155 (1988).

37. Yang, Y., Longini, I. M. \& Halloran, M. E. Design and evaluation of prophylactic interventions using infectious disease incidence data from close contact groups. Department of Biostatistics, Emory University, Technical Report 04-09 http://www.sph.emory.edu/bios/tech/Tech_Report_04-09.pdf (2004).

Supplementary Information is linked to the online version of the paper at www.nature.com/nature.

Acknowledgements We thank the National Institute of General Medical Sciences MIDAS Program (N.M.F., D.A.T.C. and D.S.B.), the Medical Research Council (N.M.F.), the Royal Society (N.M.F. and C.F.), the Howard Hughes Medical Institute (N.M.F.), the Research Fund for the Control of Infectious Diseases of the Hong Kong SAR government (S.R.) and INSERM (S.C.) for research funding. We thank F. Carrat for providing household data used in this study, and N. Cox, F. Hayden, B. Schwartz, K. Stohr and members of the MIDAS consortium for useful discussions. We thank the MIDAS informatics group for computational resources.

Author Contributions N.M.F. designed, implemented and ran the model, integrated the demographic and disease datasets used, and drafted and revised the text. All other authors edited or commented on the text. D.A.T.C. identified, collated and processed the Thai demographic and travel datasets used and provided input on model assumptions. S.C. analysed the French household dataset used to estimate key epidemiological parameters. C.F. performed analytical modelling of household transmission, which aided the verification of the simulation and gave suggestions on control strategies to be modelled. S.R. contributed to the design of some algorithms within the simulation model. A.M. assisted with collating data on Thai schools and administrative boundaries. S.I. provided feedback on the realism of model assumptions and the likely feasibility of different control options. D.S.B. provided input into model design and assumptions, advised on the presentation of results, assisted with data collection and organized meetings with stakeholders.

Author Information Reprints and permissions information is available at npg.nature.com/reprintsandpermissions. The authors declare no competing financial interests. Correspondence and requests for materials should be addressed to N.M.F. (neil.ferguson@imperial.ac.uk). 